\title{
Technoeconomic energy system data for modeling of India and the GCC countries
}

\section{Miles Weinstein ( $\nabla$ mileswei@umich.edu )}

Climate Compatible Growth, UK https://orcid.org/0000-0003-4624-6324

Youssef Almulla

Energy Department, Royal Institute of Technology (KTH), Sweden https://orcid.org/0000-0003-3015-8610

Avinash Vijay

Department of Engineering Science, University of Oxford, UK https://orcid.org/0000-0002-2636-1635

\section{Abhishek Shivakumar}

Climate Compatible Growth, UK

Will Usher

Energy Department, Royal Institute of Technology (KTH), Sweden https://orcid.org/0000-0001-9367-1791

\section{Chris Arderne}

Climate Compatible Growth, UK

\section{Adam Hawkes}

Imperial College London, UK https://orcid.org/0000-0001-9720-332X

\section{Mark Howells}

Imperial College London, UK; Loughborough University, UK https://orcid.org/0000-0001-6419-4957

\section{Data Note}

Keywords: Energy policy, Renewable energy, Cost optimisation, India, GCC

Posted Date: March 12th, 2021

DOI: https://doi.org/10.21203/rs.3.rs-320312/v1

License: @ (i) This work is licensed under a Creative Commons Attribution 4.0 International License. Read Full License 


\section{Abstract}

India has seen rapid increases in GDP, energy access, and population in recent decades, more than doubling its overall energy consumption since 2000 . Meanwhile, India produces approximately $70 \%$ of its electricity from coal. With electricity demand only projected to grow in the coming years, the Government of India has pledged to install $450 \mathrm{GW}$ of renewable energy by 2030. The Gulf Cooperation Council (GCC) countries[1], meanwhile, have comparatively small populations with excellent renewable energy resources, particularly solar. The ability to trade power between these two regions could potentially provide India with a highly reliable carbon-free power source. At the same time, it can motivate the shift to low carbon economy in the GCC and add a new market for its solar power. The provided data in this article relate to the current makeup of the energy systems of both regions, renewable resource potentials, and projections of future demand. The data have been compiled from numerous sources, mainly government and international agencies.

${ }^{[1]}$ GCC countries are Bahrain, Kuwait, Oman, Qatar, Saudi Arabia, and the United Arab Emirates

\section{Specifications Table}

\begin{tabular}{ll} 
Subject area & Energy and Sustainability \\
\hline $\begin{array}{l}\text { More specific } \\
\text { subject area }\end{array}$ & Energy resource and capacity modelling \\
\hline $\begin{array}{l}\text { Type of data } \\
\text { How data was } \\
\text { acquired }\end{array}$ & $\begin{array}{l}\text { Dables } \\
\text { agencies, research institutions, and news outlets }\end{array}$ \\
\hline Data format & Raw and analyzed \\
\hline $\begin{array}{l}\text { Experimental } \\
\text { factors }\end{array}$ & \\
\hline $\begin{array}{l}\text { Experimental } \\
\text { features }\end{array}$ & \\
\hline $\begin{array}{l}\text { Data source } \\
\text { location }\end{array}$ & India, Bahrain, Kuwait, Oman, Saudi Arabia, Qatar, United Arab Emirates \\
\hline $\begin{array}{l}\text { Data } \\
\text { accessibility }\end{array}$ & With the article and public repository: PLEXOS World 2015 https://doi.org/10.7910/DVN/CBYXBY \\
\end{tabular}

\section{Value Of The Data}

- This compilation of data provides a resource for building a techno-economic energy model of India and the Gulf Cooperation Council (GCC) countries, namely Bahrain, Kuwait, Oman, Saudi Arabia, Qatar and the United Arab Emirates (UAE).

- The data can assist in energy system planning and expansion to 2050.

- Data collection for techno-economic modelling is often a painstaking process, requiring accurate data from many sources. The data provided in this article are relevant to any energy model in the aforementioned countries, thus allowing researchers to focus on their specific research goals.

\section{Data}

The data in this paper have been compiled to assist in building a base-level energy model for India and the GCC countries. The data have been collected from various country-specific and international sources. The data include installed and 
planned generation and transmission capacity, renewable resource potentials, relevant costs, current and projected power demand, and other techno-economic parameters. Most datasets for India are region-specific. The regions used are according to regional power grids, as shown in Figure 1.

For the GCC countries, the data are on the national level except for Saudi Arabia which is split into four regions (R1: Central region, R2: Eastern region, R3: Southern Region and R4: Western Region). These four regions reflect the structure of the electricity system in Saudi Arabia and Saudi Electricity Company's (SEC's) classification as shown in Figure 2.

Table 1. Description of datasets provided in the article

\begin{tabular}{|ll|}
\hline Title & Description \\
\hline Table 2 & Installed non-renewable generation capacity in India \\
\hline Table 3 & Renewable installed capacities in India by state and region \\
\hline Table 4 & GCC installed capacity by fuel type \\
\hline Table 5 & GCC installed capacity by technology \\
\hline Table 6 & Planned coal generation capacity in India to 2025 \\
\hline Table 7 & India planned nuclear generation capacity to 2025 \\
\hline Table 8 & Planned nuclear power capacities in UAE and Saudi Arabia \\
\hline Table 9 & Renewable energy targets in GCC countries \\
\hline Table 10 & Estimated renewable resource potential in India by state and region \\
\hline Table 11 & Inter-regional transmission links in India \\
\hline Table 12 & Transmission capacity in India \\
\hline Table 13 & GCC interconnector capacity per country \\
\hline Table 14 & Annual electricity demand projection for India \\
\hline Table 15 & Annual electricity demand projection for GCC countries \\
\hline Table 16 & Capital cost of new transmission in India \\
\hline Table 17 & Availability factors \\
\hline Table 18 & Hydropower capacity factors in India \\
\hline
\end{tabular}

\section{Power generation}

\section{Non-renewable capacity in India}

Table 2 displays non-renewable power generation capacity by region as of September 30, 2020 from [3]. Large hydro refers to hydropower plants greater than $25 \mathrm{MW}$. The data do not include captive capacity.

Table 2. Installed non-renewable generation capacity in India (GW) 


\begin{tabular}{|llllll|}
\hline Region & Coal & Gas & Diesel & Nuclear & Large Hydro \\
\hline Northern Region & 53.44 & 5.78 & 0 & 1.62 & 20.04 \\
\hline Western Region & 73.74 & 10.81 & 0 & 1.84 & 7.56 \\
\hline Southern Region & 44.36 & 6.49 & 0.43 & 3.32 & 11.77 \\
\hline Eastern Region & 27.29 & 0.1 & 0 & 0 & 4.64 \\
\hline Northeastern Region & 0.77 & 1.78 & 0.04 & 0 & 1.69 \\
\hline
\end{tabular}

Renewable capacity in India

Table 3 displays installed renewable capacity in India as of December 31, 2019 (except biomass), according to [4]. Biomass capacities are as of October 31, 2020 according to [5]. Biomass includes independent power plants, bagasse cogeneration, and non-bagasse cogeneration. Small hydro includes hydropower plants below $25 \mathrm{MW}$.

Table 3. Renewable installed capacities in India by state and region (MW) 


\begin{tabular}{|c|c|c|c|c|c|}
\hline Region/State & Grid-connected Solar & Rooftop Solar & Wind & Small hydro & Biomass \\
\hline Eastern Region & 695.07 & 77.97 & 0 & 289.99 & 508.14 \\
\hline Bihar & 149.35 & 6.94 & 0 & 70.70 & 124.7 \\
\hline Jharkhand & 38.40 & 13.57 & 0 & 4.05 & 4.3 \\
\hline Odisha & 397.84 & 14.27 & 0 & 64.63 & 59.22 \\
\hline Sikkim & 0.07 & 0.07 & 0 & 52.11 & 0 \\
\hline West Bengal & 109.41 & 43.12 & 0 & 98.5 & 319.92 \\
\hline Northeast Region & 63.47 & 44.04 & 0 & 286.35 & 15.80 \\
\hline Arunachal Pradesh & 5.61 & 4.34 & 0 & 131.11 & 0 \\
\hline Assam & 41.23 & 30.56 & 0 & 34.11 & 2 \\
\hline Manipur & 4.58 & 4.55 & 0 & 5.45 & 0 \\
\hline Meghalaya & 0.12 & 0.12 & 0 & 32.53 & 13.80 \\
\hline Mizoram & 1.52 & 1.43 & 0 & 36.47 & 0 \\
\hline Nagaland & 1.00 & 0.08 & 0 & 30.67 & 0 \\
\hline Tripura & 9.41 & 2.96 & 0 & 16.01 & 0 \\
\hline Northern Region & 7646.15 & 688.45 & 4299.72 & 1597.31 & 3062.04 \\
\hline Chandigarh & 36.99 & 29.98 & 0 & 0 & 0 \\
\hline Delhi & 156.12 & 109.80 & 0 & 0 & 0 \\
\hline Haryana & 249.27 & 118.47 & 0 & 73.50 & 210.66 \\
\hline Himachal Pradesh & 32.57 & 15.46 & 0 & 906.51 & 9.20 \\
\hline Jammu \& Kashmir & 19.30 & 10.81 & 0 & 180.48 & 0 \\
\hline Punjab & 947.10 & 67.85 & 0 & 173.55 & 473.45 \\
\hline Rajasthan & 4844.21 & 119.50 & 4299.72 & 23.85 & 121.25 \\
\hline Uttar Pradesh & 1045.10 & 140.87 & 0 & 25.1 & 2117.26 \\
\hline Uttarakhand & 315.49 & 75.71 & 0 & 214.32 & 130.22 \\
\hline Southern Region & 18390.31 & 491.95 & 18321.72 & 1878.78 & 3545.99 \\
\hline Andhra Pradesh & 3559.02 & 88.03 & 4092.45 & 162.11 & 483.67 \\
\hline Karnataka & 7274.92 & 131.83 & 4753.40 & 1280.73 & 1887.3 \\
\hline Kerala & 141.75 & 41.75 & 62.50 & 222.02 & 2.27 \\
\hline Puducherry & 5.51 & 1.92 & 0 & 0 & 0 \\
\hline Tamil Nadu & 3788.36 & 155.78 & 9285.27 & 123.05 & 1012.65 \\
\hline Telangana & 3620.75 & 72.64 & 128.10 & 90.87 & 160.1 \\
\hline Western Region & 6922.6 & 582.31 & 14879.44 & 613.89 & 3013.95 \\
\hline Chhattisgarh & 231.35 & 10.39 & 0 & 76.00 & 244.9 \\
\hline
\end{tabular}




\begin{tabular}{|llllll|} 
Dadra \& Nagar Haveli & 5.46 & 0.48 & 0 & 0 & 0 \\
\hline Daman \& Diu & 16.56 & 0.39 & 0 & 0 & 0 \\
\hline Goa & 4.78 & 3.83 & 0 & 0.05 & 0 \\
\hline Gujarat & 2763.55 & 301.71 & 7359.22 & 62.35 & 77.3 \\
\hline Madhya Pradesh & 2237.48 & 49.40 & 2519.89 & 95.91 & 107.35 \\
\hline Maharashtra & 1663.42 & 216.11 & 5000.33 & 379.58 & 2584.4 \\
\hline
\end{tabular}

\section{GCC installed capacity}

The power installed capacity data were obtained from [6] and calibrated with data from official agencies in each country for the base modeling year 2015 [7]-[9]. Table 4 shows a summary of installed capacity, while Table 5 elaborates on these figures, breaking them down by technology and, in the case of Saudi Arabia, by region.

Table 4. GCC installed capacity (GW) by fuel type

\begin{tabular}{|llllllll|}
\hline Country & Crude oil & Heavy Fuel Oil & Coal & Gas & Diesel & Solar & Total \\
\hline Saudi Arabia & 19.42 & 20.93 & 0 & 57.91 & 8.61 & 0 & 106.87 \\
\hline UAE & 0 & 0.31 & 0 & 28.35 & 0.03 & 0.06 & 28.75 \\
\hline Qatar & 0 & 0 & 0 & 8.56 & 0 & 0 & 8.56 \\
\hline Oman & 0 & 0 & 0 & 7.74 & 0 & 0 & 7.74 \\
Kuwait & 8.75 & 0 & 0 & 8.43 & 0 & 0 & 17.18 \\
\hline Bahrain & 0 & 0 & 0 & 3.92 & 0 & 0 & 3.92 \\
\hline
\end{tabular}

Table 5. GCC installed capacity (GW) by technology 


\begin{tabular}{|c|c|c|c|c|}
\hline Country & Region & Fuel & Technology & Capacity \\
\hline \multirow[t]{7}{*}{ UAE } & \multirow[t]{7}{*}{ National } & Heavy Fuel & steam cycle & 0.31 \\
\hline & & Natural gas & steam cycle & 1.78 \\
\hline & & Natural gas & open cycle gas turbine & 5.64 \\
\hline & & Natural gas & Combined cycle & 20.93 \\
\hline & & Diesel & Diesel Engine & 0.03 \\
\hline & & Solar & Solar Thermal (CSP) & 0.06 \\
\hline & & \multicolumn{2}{|c|}{ Total Capacity } & 28.76 \\
\hline \multirow[t]{26}{*}{ Saudi Arabia } & \multirow[t]{10}{*}{ Central Region } & Heavy Fuel & steam cycle & 0.35 \\
\hline & & Heavy Fuel & open cycle gas turbine & 0.6 \\
\hline & & Natural gas & open cycle gas turbine & 4.97 \\
\hline & & Natural gas & Combined cycle & 1.2 \\
\hline & & Crude Oil & steam cycle & 0.46 \\
\hline & & Crude Oil & open cycle gas turbine & 7.89 \\
\hline & & Crude Oil & combined cycle & 1.65 \\
\hline & & Diesel & steam cycle & 0.35 \\
\hline & & Diesel & open cycle gas turbine & 1.04 \\
\hline & & Diesel & Diesel Engine & 0.02 \\
\hline & \multirow[t]{8}{*}{ Eastern Region } & Heavy Fuel & steam cycle & 0.62 \\
\hline & & Heavy Fuel & Diesel Engine & 0.07 \\
\hline & & Natural gas & steam cycle & 13.45 \\
\hline & & Natural gas & open cycle gas turbine & 8.71 \\
\hline & & Natural gas & Combined cycle & 8.42 \\
\hline & & Crude Oil & open cycle gas turbine & 0.68 \\
\hline & & Diesel & open cycle gas turbine & 1.16 \\
\hline & & Diesel & Diesel Engine & 0.11 \\
\hline & \multirow[t]{4}{*}{ Southern Region } & Crude Oil & steam cycle & 1.02 \\
\hline & & Crude Oil & open cycle gas turbine & 1.11 \\
\hline & & Diesel & open cycle gas turbine & 2.83 \\
\hline & & Diesel & Diesel Engine & 0.22 \\
\hline & \multirow[t]{4}{*}{ Western Region } & Heavy Fuel & steam cycle & 18.49 \\
\hline & & Heavy Fuel & open cycle gas turbine & 0.8 \\
\hline & & Natural gas & open cycle gas turbine & 0.23 \\
\hline & & Crude Oil & steam cycle & 1.57 \\
\hline
\end{tabular}

Page $7 / 25$ 


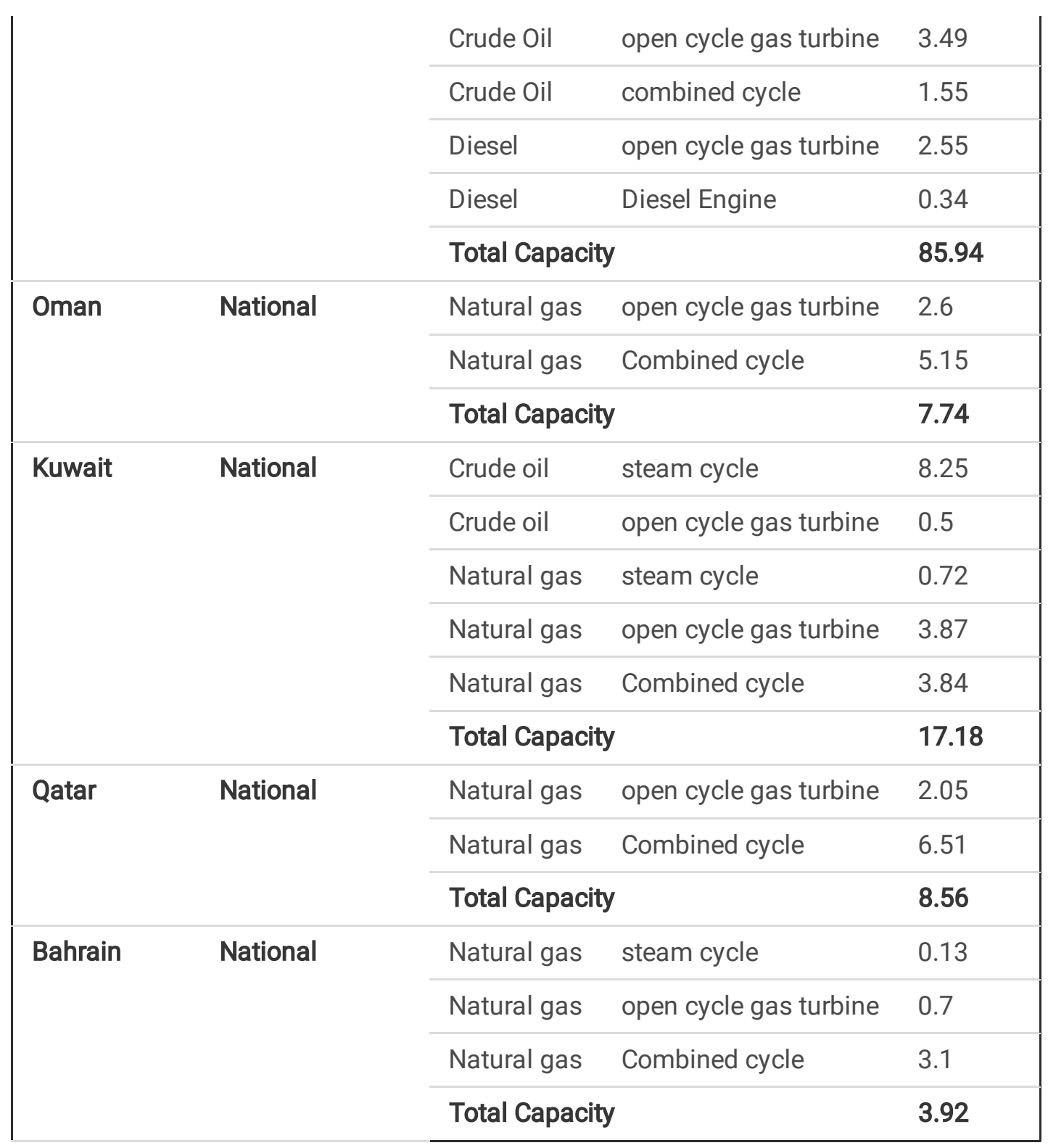

\section{Planned capacity}

Table 6 shows new coal power plant capacity in India expected to go online in each region by 2025 according to [10]-[32]. Due to data gaps, some expected years of commissioning have been assumed. In these cases, which have been marked with an asterisk in Table 6, planned capacity can be considered to be allocated to a specific year. Total values of planned capacity to 2025 remain accurate (i.e. without regard to commissioning year).

Table 6. Annual planned coal generation capacity in India to 2025 (GW) 


\begin{tabular}{|llllll|}
\hline $\begin{array}{l}\text { Expected Commissioning } \\
\text { Year }\end{array}$ & $\begin{array}{l}\text { Eastern } \\
\text { Region }\end{array}$ & $\begin{array}{l}\text { Northeastern } \\
\text { Region }\end{array}$ & $\begin{array}{l}\text { Northern } \\
\text { Region }\end{array}$ & $\begin{array}{l}\text { Southern } \\
\text { Region }\end{array}$ & $\begin{array}{l}\text { Western } \\
\text { Region }\end{array}$ \\
\hline 2020 & 3.58 & 0 & 0 & 0 & 1.60 \\
\hline 2021 & $1.98^{\star}$ & 0 & $7.26^{*}$ & $8.92^{*}$ & 1.40 \\
\hline 2022 & $5.32^{\star}$ & 0 & 1.32 & 0 & $2.80^{*}$ \\
\hline 2023 & 0 & 0 & 1.40 & 3.60 & 0 \\
\hline 2024 & 0 & 0 & 0 & 0 & 0 \\
\hline 2025 & 0 & 0 & 1.40 & 0 & 0 \\
\hline Total & 10.88 & 0 & 11.38 & 12.52 & 5.8 \\
\hline
\end{tabular}

Table 7 displays all planned nuclear capacity to 2025, according to [33]-[37]. Each value is a capacity addition in the given year and region.

Table 7. Planned nuclear generation capacity to 2025 in India (GW)

\begin{tabular}{|llllll|}
\hline $\begin{array}{l}\text { Expected Commissioning } \\
\text { Year }\end{array}$ & $\begin{array}{l}\text { Eastern } \\
\text { Region }\end{array}$ & $\begin{array}{l}\text { Northeastern } \\
\text { Region }\end{array}$ & $\begin{array}{l}\text { Northern } \\
\text { Region }\end{array}$ & $\begin{array}{l}\text { Southern } \\
\text { Region }\end{array}$ & $\begin{array}{l}\text { Western } \\
\text { Region }\end{array}$ \\
\hline 2020 & 0 & 0 & 0 & 0 & 0 \\
\hline 2021 & 0 & 0 & 0 & 0.5 & 1.4 \\
\hline 2022 & 0 & 0 & 0 & 0 & 0 \\
\hline 2023 & 0 & 0 & 1.4 & 2 & 0 \\
\hline 2024 & 0 & 0 & 0 & 0 & 0 \\
\hline 2025 & 0 & 0 & 1.4 & 2 & 0 \\
\hline
\end{tabular}

Table 8 displays all planned nuclear capacity in the GCC with annual additions to 2025, according to [38], [39].

Table 8. Planned nuclear power annual capacity addition in UAE and Saudi Arabia (GW)

\begin{tabular}{|lll|}
\hline Expected Commissioning Year & UAE & SA \\
\hline 2020 & 0 & 0 \\
\hline 2021 & 1.4 & 0 \\
\hline 2022 & 1.4 & 0 \\
2023 & 1.4 & 0 \\
\hline 2024 & 1.4 & 0 \\
2025 & 0 & 0 \\
\hline 2040 & 0 & 18 \\
\hline
\end{tabular}

Table 9. Renewable energy targets in GCC countries 


\begin{tabular}{|llr|}
\hline Country & Target & Year \\
\hline KSA & $9.5 \mathrm{GW}$ of Renewables & 2022 \\
\hline KSA & $54 \mathrm{GW}$ of renewables (41 GW solar, 9 GW Wind, 3 GW W2E and 1 GW Geo) & 2040 \\
\hline UAE & $24 \%$ clean energy & 2021 \\
\hline UAE & $7 \%$ of capacity ( 5 GW solar PV) & 2030 \\
\hline Qatar & $20 \%$ of capacity $(1.8 \mathrm{GW})$ & 2030 \\
\hline Kuwait & $5 \%$ of generation & 2020 \\
\hline Kuwait & $15 \%$ of generation $(5.7 \mathrm{GW}$ CSP, $4.6 \mathrm{GW} \mathrm{PV} \mathrm{and} \mathrm{0.7} \mathrm{GW} \mathrm{Wind)}$ \\
\hline Bahrain & $5 \%$ of capacity & 2020 \\
\hline
\end{tabular}

\section{Renewable Resources in India}

Table 10 displays renewable resource potentials in India aggregated by region [41].

Table 10. Estimated renewable resource potential in India by state and region (MW) 


\begin{tabular}{|c|c|c|c|c|c|c|}
\hline Region/State & $\begin{array}{l}\text { Waste to } \\
\text { Energy }\end{array}$ & $\begin{array}{l}\text { Biomass } \\
\text { power }\end{array}$ & $\begin{array}{l}\text { Wind @ } \\
\text { 100m }\end{array}$ & $\begin{array}{l}\text { Small } \\
\text { hydro }\end{array}$ & $\begin{array}{l}\text { Cogeneration } \\
\text { (bagasse) }\end{array}$ & Solar \\
\hline Eastern Region & 253 & 1353 & 3095 & 1700 & 300 & 67879 \\
\hline Bihar & 73 & 619 & 0 & 527 & 300 & 12719 \\
\hline Jharkhand & 10 & 90 & 0 & 228 & 0 & 18180 \\
\hline Odisha & 22 & 246 & 3093 & 286 & 0 & 25780 \\
\hline Sikkim & 0 & 2 & 0 & 267 & 0 & 4940 \\
\hline West Bengal & 148 & 396 & 2 & 392 & 0 & 6260 \\
\hline Northeast Region & 16 & 258 & 0 & 2995 & 0 & 57782 \\
\hline $\begin{array}{l}\text { Arunachal } \\
\text { Pradesh }\end{array}$ & 0 & 8 & 0 & 2065 & 0 & 8650 \\
\hline Assam & 8 & 212 & 0 & 202 & 0 & 14182 \\
\hline Manipur & 2 & 13 & 0 & 100 & 0 & 10630 \\
\hline Meghalaya & 2 & 11 & 0 & 230 & 0 & 5860 \\
\hline Mizoram & 2 & 1 & 0 & 169 & 0 & 9090 \\
\hline Nagaland & 0 & 10 & 0 & 182 & 0 & 7290 \\
\hline Tripura & 2 & 3 & 0 & 47 & 0 & 2080 \\
\hline Northern Region & 451 & 3159 & 18770 & 8029 & 1900 & 336250 \\
\hline Chandigarh & 6 & 0 & 0 & 0 & 0 & 0 \\
\hline Delhi & 131 & 0 & 0 & 0 & 0 & 2050 \\
\hline Haryana & 24 & 1333 & 0 & 107 & 350 & 4560 \\
\hline Himachal Pradesh & 2 & 142 & 0 & 3460 & 0 & 33840 \\
\hline Jammu \& Kashmir & 0 & 43 & 0 & 1707 & 0 & 111050 \\
\hline Punjab & 45 & 0 & 0 & 578 & 300 & 2810 \\
\hline Rajasthan & 62 & 0 & 18770 & 52 & 0 & 142310 \\
\hline Uttar Pradesh & 176 & 1617 & 0 & 461 & 1250 & 22830 \\
\hline Uttarakhand & 5 & 24 & 0 & 1664 & 0 & 16800 \\
\hline Southern Region & 313 & 3823 & 139983 & 5488 & 1200 & 107330 \\
\hline Andhra Pradesh & 123 & 578 & 44229 & 409 & 300 & 38440 \\
\hline Karnataka & 0 & 1131 & 55857 & 3726 & 450 & 24700 \\
\hline Kerala & 36 & 1044 & 1700 & 647 & 0 & 6110 \\
\hline Puducherry & 3 & 0 & 153 & 0 & 0 & 0 \\
\hline Tamil Nadu & 151 & 1070 & 33800 & 604 & 450 & 17670 \\
\hline Telangana & 0 & 0 & 4244 & 102 & 0 & 20410 \\
\hline Western Region & 501 & 4734 & 140387 & 2911 & 1600 & 180900 \\
\hline
\end{tabular}




\begin{tabular}{|lllllll|}
\hline Chhattisgarh & 24 & 236 & 77 & 1098 & 0 & 18270 \\
\hline $\begin{array}{l}\text { Dadra \& Nagar } \\
\text { Haveli }\end{array}$ & 0 & 0 & 0 & 0 & 0 & 0 \\
\hline Daman \& Diu & 0 & 0 & 0 & 0 & 0 & 0 \\
\hline Goa & 0 & 26 & 1 & 5 & 0 & 880 \\
\hline Gujarat & 112 & 1221 & 84431 & 202 & 350 & 35770 \\
\hline Madhya Pradesh & 78 & 1364 & 10484 & 820 & 0 & 61660 \\
\hline Maharashtra & 287 & 1887 & 45394 & 786 & 1250 & 64320 \\
\hline
\end{tabular}

\section{Transmission}

Table 11 displays transmission capacity between India's regional grids as reported by the National Electricity Plan (NEP), 2017-2022 [42].

Table 11. Inter-regional transmission links in India (GW)

\begin{tabular}{|llll|}
\hline Inter-regional corridors & Capacity in 2017 & Addition expected during 2017-2022 & Required by 2021-22 \\
\hline West-North & 15.42 & 21.3 & 36.72 \\
\hline Northeast-North & 3 & 0 & 3 \\
\hline East-North & 21.03 & 1.5 & 22.53 \\
\hline East-West & 12.79 & 8.4 & 21.19 \\
\hline East-South & 7.83 & 0 & 7.83 \\
\hline West-South & 12.12 & 11.8 & 23.92 \\
\hline East-Northeast & 2.86 & 0 & 2.86 \\
\hline Total & $\mathbf{7 5 . 0 5}$ & $\mathbf{4 3}$ & $\mathbf{1 1 8 . 0 5}$ \\
\hline
\end{tabular}

Installed transmission capacity for all of India was obtained from India's NEP [42]. Regional transmission capacities listed below have been inferred based on the proportion of installed generation capacity in each region, and should be used for reference purposes only [3]. Country-level data remain accurate.

Table 12. Transmission capacity in India (GW) 


\begin{tabular}{|llll|}
\hline Region & Capacity in 2017 & Addition expected during 2017-2022 & Required by 2021-22 \\
\hline All India & 721.27 & 383.69 & 1104.96 \\
\hline Northern Region & 193.08 & 102.71 & 295.79 \\
\hline Western Region & 236.41 & 125.76 & 362.17 \\
\hline Southern Region & 217.74 & 115.83 & 333.58 \\
\hline Eastern Region & 64.92 & 34.54 & 99.46 \\
\hline Northeastern Region & 8.97 & 4.77 & 13.74 \\
\hline
\end{tabular}

The GCC countries are connected with each other through the GCC interconnector. The transmission capacity of the interconnector varies between countries and the following table shows the existing capacity of each trans-border line [43], [44]:

Table 13. GCC interconnector capacity per country

\begin{tabular}{|ll|}
\hline Interconnector & Capacity (MW) \\
\hline Kuwait - GCC $^{[2]}$ & 1200 \\
\hline KSA - GCC & 1200 \\
\hline UAE - GCC & 900 \\
\hline Oman - UAE & 400 \\
\hline Qatar - GCC & 750 \\
\hline Bahrain - GCC & 600 \\
\hline
\end{tabular}

\section{Demand}

Table 14 shows an annual demand projection for India and each region, with the projected Compound Annual Growth Rate (CAGR) for All-India in each year.

Table 14. India annual electricity demand projection, Business as usual scenario (PJ)

Sources: Own elaboration using data from [45]-[48] 


\begin{tabular}{|c|c|c|c|c|c|c|c|}
\hline Year & All-India ${ }^{[3]}$ & $\begin{array}{l}\text { Eastern } \\
\text { Region }\end{array}$ & $\begin{array}{l}\text { Northeast } \\
\text { Region }\end{array}$ & $\begin{array}{l}\text { Northern } \\
\text { Region }\end{array}$ & $\begin{array}{l}\text { Southern } \\
\text { Region }\end{array}$ & $\begin{array}{l}\text { Western } \\
\text { Region }\end{array}$ & $\begin{array}{l}\text { CAGR (All- } \\
\text { India) }\end{array}$ \\
\hline 2015 & 4011.87 & 460.33 & 53.47 & 1243.94 & 1044.38 & 1208.58 & \\
\hline 2016 & 4114.54 & 472.11 & 54.84 & 1275.78 & 1071.10 & 1239.51 & \\
\hline 2017 & 4367.97 & 520.86 & 57.13 & 1361.71 & 1137.13 & 1289.89 & \\
\hline 2018 & 4588.43 & 561.68 & 59.36 & 1433.84 & 1193.54 & 1338.67 & \\
\hline 2019 & 4647.64 & 580.10 & 59.73 & 1455.71 & 1208.81 & 1341.93 & \\
\hline 2020 & 4415.25 & 560.16 & 56.46 & 1386.37 & 1148.80 & 1262.17 & $-5 \%$ \\
\hline 2021 & 4649.26 & 598.82 & 59.17 & 1463.75 & 1210.42 & 1315.76 & $5.3 \%$ \\
\hline 2022 & 4863.13 & 635.66 & 61.58 & 1535.21 & 1266.87 & 1362.40 & $4.6 \%$ \\
\hline 2023 & 5111.15 & 677.91 & 64.37 & 1617.80 & 1332.25 & 1417.34 & $5.1 \%$ \\
\hline 2024 & 5371.82 & 722.88 & 67.25 & 1704.59 & 1400.86 & 1474.70 & $5.1 \%$ \\
\hline 2025 & 5645.78 & 770.64 & 70.22 & 1795.73 & 1472.85 & 1534.72 & $5.1 \%$ \\
\hline 2026 & 5933.71 & 821.24 & 73.28 & 1891.51 & 1548.52 & 1597.45 & $5.1 \%$ \\
\hline 2027 & 6210.23 & 871.17 & 76.16 & 1983.91 & 1621.16 & 1656.02 & $4.66 \%$ \\
\hline 2028 & 6499.62 & 923.82 & 79.15 & 2080.70 & 1697.11 & 1716.96 & $4.66 \%$ \\
\hline 2029 & 6802.50 & 979.32 & 82.26 & 2182.10 & 1776.50 & 1780.36 & $4.66 \%$ \\
\hline 2030 & 7119.50 & 1037.81 & 85.49 & 2288.31 & 1859.47 & 1846.35 & $4.66 \%$ \\
\hline 2031 & 7451.27 & 1099.46 & 88.85 & 2399.57 & 1946.18 & 1915.03 & $4.66 \%$ \\
\hline 2032 & 7798.50 & 1164.42 & 92.35 & 2516.24 & 2036.88 & 1986.32 & $4.66 \%$ \\
\hline 2033 & 8161.91 & 1232.96 & 96.01 & 2638.58 & 2131.72 & 2060.25 & $4.66 \%$ \\
\hline 2034 & 8542.25 & 1305.34 & 99.81 & 2766.87 & 2230.84 & 2136.87 & $4.66 \%$ \\
\hline 2035 & 8940.32 & 1381.81 & 103.78 & 2901.39 & 2334.44 & 2216.25 & $4.66 \%$ \\
\hline 2036 & 9356.94 & 1462.70 & 107.92 & 3042.45 & 2442.67 & 2298.41 & $4.66 \%$ \\
\hline 2037 & 9778.00 & 1528.53 & 112.78 & 3179.36 & 2552.59 & 2401.84 & $4.5 \%$ \\
\hline 2038 & 10218.01 & 1597.31 & 117.86 & 3322.43 & 2667.45 & 2509.92 & $4.5 \%$ \\
\hline 2039 & 10677.83 & 1669.19 & 123.16 & 3471.94 & 2787.49 & 2622.87 & $4.5 \%$ \\
\hline 2040 & 11158.33 & 1744.30 & 128.70 & 3628.18 & 2912.92 & 2740.90 & $4.5 \%$ \\
\hline 2041 & 11660.45 & 1822.80 & 134.49 & 3791.45 & 3044.01 & 2864.24 & $4.5 \%$ \\
\hline 2042 & 12185.17 & 1904.82 & 140.55 & 3962.07 & 3180.99 & 2993.13 & $4.5 \%$ \\
\hline 2043 & 12733.51 & 1990.54 & 146.87 & 4140.36 & 3324.13 & 3127.82 & $4.5 \%$ \\
\hline 2044 & 13306.51 & 2080.11 & 153.48 & 4326.67 & 3473.72 & 3268.57 & $4.5 \%$ \\
\hline 2045 & 13905.31 & 2173.72 & 160.39 & 4521.37 & 3630.03 & 3415.66 & $4.5 \%$ \\
\hline 2046 & 14531.04 & 2271.54 & 167.60 & 4724.84 & 3793.38 & 3569.36 & $4.5 \%$ \\
\hline
\end{tabular}




\begin{tabular}{|llllllll|}
$\mathbf{2 0 4 7}$ & 15184.94 & 2373.75 & 175.14 & 4937.45 & 3964.09 & 3729.98 & $4.5 \%$ \\
\hline $\mathbf{2 0 4 8}$ & 15868.26 & 2480.57 & 183.03 & 5159.64 & 4142.47 & 3897.83 & $4.5 \%$ \\
\hline $\mathbf{2 0 4 9}$ & 16582.34 & 2592.20 & 191.26 & 5391.82 & 4328.88 & 4073.23 & $4.5 \%$ \\
\hline $\mathbf{2 0 5 0}$ & 17328.54 & 2708.85 & 199.87 & 5634.46 & 4523.68 & 4256.53 & $4.5 \%$ \\
\hline
\end{tabular}

\section{GCC Annual Electricity Demand Projections, Business as Usual Scenario}

The electricity demand projections are based on the historical growth in demand in each of the GCC countries obtained from official reports [7]-[9], [49]-[57]. Further explanation of the approach is given in the 'Experimental Design, Material and Methods' section of the report.

Table 15. Annual electricity demand projection for GCC countries (PJ) 


\begin{tabular}{|c|c|c|c|c|c|c|c|c|c|}
\hline Year & KSA-R1 & KSA-R2 & KSA-R3 & KSA-R4 & UAE & OMN & QTR & KWT & BHN \\
\hline 2015 & 285.6 & 283.7 & 96.0 & 310.5 & 458.51 & 112.66 & 149.40 & 245.83 & 62.1 \\
\hline 2016 & 291.0 & 289.1 & 97.8 & 316.4 & 466.55 & 114.99 & 152.30 & 252.30 & 61.4 \\
\hline 2017 & 296.4 & 294.4 & 99.6 & 322.3 & 484.39 & 124.45 & 164.00 & 262.03 & 64.8 \\
\hline 2018 & 301.9 & 299.8 & 101.5 & 328.2 & 498.70 & 129.43 & 172.49 & 267.43 & 64.9 \\
\hline 2019 & 307.3 & 305.2 & 103.3 & 334.1 & 513.43 & 134.61 & 177.24 & 272.94 & 65.8 \\
\hline 2020 & 312.7 & 310.6 & 105.1 & 340.0 & 528.59 & 139.99 & 182.13 & 278.56 & 66.6 \\
\hline 2021 & 318.1 & 316.0 & 106.9 & 345.9 & 544.21 & 145.59 & 187.15 & 284.29 & 67.5 \\
\hline 2022 & 323.7 & 321.5 & 108.8 & 352.0 & 560.28 & 151.41 & 192.30 & 290.15 & 68.4 \\
\hline 2023 & 329.4 & 327.2 & 110.7 & 358.1 & 576.83 & 157.47 & 197.61 & 296.12 & 69.3 \\
\hline 2024 & 335.1 & 332.9 & 112.6 & 364.4 & 593.87 & 163.77 & 203.05 & 302.22 & 70.3 \\
\hline 2025 & 341.0 & 338.7 & 114.6 & 370.8 & 611.42 & 170.32 & 208.65 & 308.45 & 71.2 \\
\hline 2026 & 347.0 & 344.6 & 116.6 & 377.3 & 629.48 & 177.13 & 214.40 & 314.80 & 72.1 \\
\hline 2027 & 353.0 & 350.7 & 118.7 & 383.9 & 648.07 & 184.22 & 220.31 & 321.28 & 73.1 \\
\hline 2028 & 359.2 & 356.8 & 120.7 & 390.6 & 667.22 & 191.59 & 226.38 & 327.90 & 74.1 \\
\hline 2029 & 365.5 & 363.1 & 122.9 & 397.4 & 686.92 & 199.25 & 232.63 & 334.65 & 75.1 \\
\hline 2030 & 371.9 & 369.4 & 125.0 & 404.4 & 707.22 & 207.22 & 239.04 & 341.54 & 76.1 \\
\hline 2031 & 376.8 & 374.3 & 126.7 & 409.7 & 728.11 & 215.51 & 245.63 & 348.57 & 77.1 \\
\hline 2032 & 382.7 & 380.1 & 128.6 & 416.1 & 749.61 & 224.13 & 252.40 & 355.75 & 78.1 \\
\hline 2033 & 388.6 & 386.0 & 130.6 & 422.5 & 771.76 & 233.09 & 259.36 & 363.08 & 79.2 \\
\hline 2034 & 394.4 & 391.8 & 132.6 & 428.9 & 794.56 & 242.42 & 266.50 & 370.55 & 80.2 \\
\hline 2035 & 400.3 & 397.6 & 134.5 & 435.3 & 818.03 & 252.11 & 273.85 & 378.18 & 81.3 \\
\hline 2036 & 406.2 & 403.5 & 136.5 & 441.6 & 842.19 & 262.20 & 281.40 & 385.97 & 82.4 \\
\hline 2037 & 412.1 & 409.3 & 138.5 & 448.0 & 867.07 & 272.69 & 289.16 & 393.92 & 83.5 \\
\hline 2038 & 417.9 & 415.1 & 140.5 & 454.4 & 892.68 & 283.59 & 297.13 & 402.03 & 84.6 \\
\hline 2039 & 423.8 & 421.0 & 142.4 & 460.8 & 919.05 & 294.94 & 305.32 & 410.31 & 85.7 \\
\hline 2040 & 429.7 & 426.8 & 144.4 & 467.2 & 946.20 & 306.73 & 313.73 & 418.76 & 86.8 \\
\hline 2041 & 435.5 & 432.6 & 146.4 & 473.6 & 974.15 & 319.00 & 322.38 & 427.38 & 88.0 \\
\hline 2042 & 441.4 & 438.5 & 148.4 & 479.9 & 1002.92 & 331.76 & 331.27 & 436.18 & 89.2 \\
\hline 2043 & 447.3 & 444.3 & 150.3 & 486.3 & 1032.55 & 345.03 & 340.40 & 445.16 & 90.3 \\
\hline 2044 & 453.1 & 450.1 & 152.3 & 492.7 & 1063.05 & 358.84 & 349.78 & 454.33 & 91.6 \\
\hline 2045 & 459.0 & 456.0 & 154.3 & 499.1 & 1094.45 & 373.19 & 359.43 & 463.69 & 92.8 \\
\hline 2046 & 464.9 & 461.8 & 156.3 & 505.5 & 1126.78 & 388.12 & 369.33 & 473.24 & 94.0 \\
\hline 2047 & 470.8 & 467.6 & 158.2 & 511.9 & 1160.07 & 403.64 & 379.51 & 482.98 & 95.3 \\
\hline
\end{tabular}




\begin{tabular}{|llllllllll|}
$\mathbf{2 0 4 8}$ & 476.6 & 473.4 & 160.2 & 518.2 & 1194.34 & 419.79 & 389.98 & 492.93 & 96.5 \\
\hline $\mathbf{2 0 4 9}$ & 482.5 & 479.3 & 162.2 & 524.6 & 1229.62 & 436.58 & 400.73 & 503.08 & 97.8 \\
\hline $\mathbf{2 0 5 0}$ & 488.4 & 485.1 & 164.1 & 531.0 & 1265.94 & 454.04 & 411.77 & 513.44 & 99.1 \\
\hline
\end{tabular}

\section{Miscellaneous Data}

Table 16 displays the unit cost of transmission based on the expected cost of capacity for the NEP [42].

Table 16. Capital cost of new transmission in India

\begin{tabular}{|l|}
\hline Cost (USD/kW) \\
839 \\
\hline
\end{tabular}

The availability factors presented below are global values, with the exception of conventional nuclear power, which shows a historical value for India.

Table 17. Availability factors

\begin{tabular}{|lll|}
\hline Technology & Value & Source \\
\hline Coal-fired power plant & 0.92 & {$[58]$} \\
\hline Open cycle gas-fired power plant & 0.92 & {$[59]$} \\
\hline Combined cycle gas-fired power plant & 0.92 & {$[59]$} \\
\hline Diesel generator & 0.92 & {$[60]$} \\
\hline Nuclear power plant & 0.95 & {$[61]$} \\
\hline Pumped storage hydropower plant & 0.98 & {$[62]$} \\
\hline Small hydropower plant (SHP) & 0.98 & {$[62]$} \\
\hline Biomass cogeneration power plant & 0.93 & {$[63]$} \\
\hline Geothermal & 0.9 & {$[64]$} \\
\hline Waste to Energy & 0.62 & {$[65]$} \\
\hline
\end{tabular}

Table 18. Hydropower capacity factors in India

Source: [66] 


\begin{tabular}{|ll|}
\hline Season & Season Average (\%) \\
\hline Winter, Dec.-Feb. & 12.24 \\
\hline Pre-monsoon, Mar.-May & 21.09 \\
\hline Monsoon, Jun.-Sep. & 65.53 \\
\hline Post-monsoon, Oct.-Nov. & 26.81 \\
\hline
\end{tabular}

\section{Additional Data}

Examples of necessary data not listed above include demand profile, capacity factors, capital costs, fixed costs, variable costs, and operational life. These can be obtained directly from the PLEXOS World 2015 dataset, accessible at the following link: https://dataverse.harvard.edu/dataset.xhtml?persistentld=doi:10.7910/DVN/CBYXBY

\section{Global Data}

Additional necessary data have been obtained from sources that are not location-specific, namely for power plant availability factors and emission ratios. Average availability factors for several technologies can be obtained from IEAETSAP. Emission factors were sourced from WRI GHG Emission Factors Compilation.

${ }^{[2]}$ Kuwait, Bahrain, Qatar and UAE are interconnected through Saudi Arabia which hosts the main GCC interconnector. Oman is connected to GCCIA through the UAE grid.

${ }^{[3]}$ All-India demand includes Lakshadweep and Andaman \& Nicobar Islands

\section{Experimental Design, Material, And Methods}

Data for India's energy system were collected mainly from reports by government agencies, namely Ministry of Power (MoP), Ministry of New and Renewable Energy (MNRE), Central Electricity Authority (CEA), and Ministry of Statistics and Program Implementation (MOSPI). Other data sources include The Energy and Resources Institute (TERI), International Energy Agency (IEA), World Resources Institute (WRI), and numerous local news publications.

Non-renewable power generation capacity by region (Table 2) was obtained from CEA [3]. Large hydropower is not considered among renewable capacity, while small hydropower is considered renewable. Renewable capacities (Table 3) were obtained from MNRE [4] and aggregated by region. Installed and planned transmission capacities (Tables 11 and 12) were obtained from the MoP National Electricity Plan (NEP), Volume II Transmission [42]. Renewable resource potentials were obtained from MOSPI Energy Statistics 2020 [41] and aggregated by region.

Capital cost of transmission in India (Table 16) was calculated based on the projected cost of installing new capacity as dictated by the NEP [42] during the 2017-2022 period.

Hydropower capacity factors for India (Table 18) were synthesized from monthly 15-year average capacity utilization for over 200 hydropower plants in India from the PLEXOS World dataset [66].

The electricity demand projection for India in Table 14 was developed considering the effect of COVID-19 lockdowns in 2020. Historical demand data to 2019 was obtained from CEA [48]. The framework for the projection was obtained from 
CEA's Long Term Electricity Demand Forecasting Report Partial Adjustment Model (PAM) [45], with adjustments being made to reflect the effects of the pandemic. Using the relationship between GDP growth and demand growth from PAM as a reference, a 5\% year-on-year (YoY) decrease in demand was assumed for 2020 based on an estimated 7.7\% drop in GDP [46]. In 2021, the IMF predicts India's GDP to jump 11.5\%, and 6.8\% in 2022 [47]. Demand increases of 5.3\% and 4.6\% have been assumed for 2021 and 2022, respectively. Thereafter, the projection uses Compound Annual Growth Rates (CAGR) of $5.1 \%$ to 2026 and $4.66 \%$ to 2036, according to the PAM's Business As Usual scenario with 7.3\% annual GDP growth. From 2037-2050 the CAGR is assumed to decrease slightly to $4.5 \%$ due to continued decoupling of GDP from energy use.

To determine the demand by region, the All-India projection described above was split according to the regional proportions of total demand projected in the PAM. Because the PAM only includes years 2016-2036, year 2015 uses the regional breakdown ratio for 2016, and years 2037-2050 use the ratio for 2036.

Data for the GCC energy system were collected mainly from the utilities and institutes in each country as well as the publicly available datasets such as PLATTS database [6]. The latest reports by the GCC government agencies were also revised. To name a few examples: the ministry of energy in Saudi Arabia (moenergy.gov.sa), the ministry of energy and Infrastructure in the UAE (moei.ae), Oman Power and Water Procurement Company (omanpwp.om), Qatar General Electricity and Water Corporation "KAHRAMAA" (km.qa).

The residual capacities in each GCC country were compiled using the PLATTS database then calibrated with the data from local ministry and/or electricity utility in each country (Table 4 and Table 5). The power infrastructure in the GCC consists mainly of thermal power plants since the contribution of renewables is still insignificant in the GCC. However, each country has its own sustainability roadmap which in some cases (i.e UAE) is translated into specific capacity targets of renewable installations in the coming years (Table 9).

The techno-economic characteristics of the power plants were based on the average values obtained from four subregions in Saudi Arabia [67]. This dataset was complemented by data from international sources such as IEA-ETSAP. The same values were assumed for the other GCC countries since no country-specific data could be found from publicly available sources.

The data for the existing GCC interconnector were obtained from the GCC Interconnection Authority [6], [7]. This includes mainly the transmission capacity in each interconnector as shown in Table 13.

The electricity demand projections for the GCC countries were developed based on historical demand data and future projections obtained from local utilities in the six countries. For example in the case of the UAE, the historical data obtained from the Ministry of Energy and Infrastructure shows an average annual growth of electricity demand by $6 \%$ from 2008-2017 [8]. A moderate or a conservative growth of electricity demand was assumed for the period of $2018-2050$ at $3 \%$. Which is half the historical growth and it is close to the growth rate assumed by Abu Dhabi utility. According to Oman's power utility (OPWP), the average annual growth in electricity demand was at a rate of $8 \%$ during 2011-2017 reaching a level of 125 PJ in 2017 [55], [68]. An annual average growth of 4\% assumed for the period 2018-2050. A similar approach was followed for the other countries (Bahrain, Kuwait and Qatar). For the case of KSA, electricity demand projections were segregated by the four regions to match the existing power infrastructure. The electricity demand for the period 2019-2030 was based on the projections developed in the study [67] then extrapolated for the other years.

\section{Declarations}

\section{Acknowledgements}

Funding was provided by The Energy and Economic Growth (EEG) and Climate Compatible Growth Program (\#CCG) of the UK's Foreign Development and Commonwealth Office (FCDO). The views expressed in this paper do not necessarily reflect 


\section{Conflict of interest}

The authors declare that they have no known competing financial interests or personal relationships that could have appeared to influence the work reported in this paper.

\section{References}

[1] A. Rose, I. Chernyakhovskiy, D. Palchak, S. Koebrich, and M. Joshi, 'Least-Cost Pathways for India's Electric Power Sector', National Renewable Energy Laboratory, Golden, Colorado, NREL/TP-6A20-76153. [Online]. Available: https://www.nrel.gov/docs/fy20osti/76153.pdf.

[2] J. I. Mikayilov, A. Darandary, R. Alyamani, F. J. Hasanov, and H. Alatawi, 'Regional heterogeneous drivers of electricity demand in Saudi Arabia: Modeling regional residential electricity demand', Energy Policy, vol. 146, p. 111796, Nov. 2020, doi: 10.1016/j.enpol.2020.111796.

[3] CEA, 'All India Installed Capacity (in MW) of Power Stations (As on 30.09.2020) (Utilities)', Central Electricity Authority, India, Sep. 2020. Accessed: Feb. 11, 2020. [Online]. Available: https://cea.nic.in/wpcontent/uploads/installed/2020/09/installed_capacity.pdf.

[4] MNRE, 'Annual Report 2019-20', Indian Ministry of New and Renewable Energy, 2020. [Online]. Available: https://mnre.gov.in/img/documents/uploads/file_f-1597797108502.pdf.

[5] MNRE, 'Bio Energy', Ministry of New and Renewable Energy. https://mnre.gov.in/bio-energy/current-status (accessed Feb. 16, 2021).

[6] Platts, 'World Electric Power Plants Database', 2015. https://www.platts.com/products/world-electric-power-plantsdatabase (accessed Nov. 29, 2017).

[7] S. E. C. SEC, 'SEC- Annual Report 2019', Riyadh, 2020. [Online]. Available: https://www.se.com.sa/enus/Pages/AnnualReports.aspx.

[8] M. of E. and I. in U. MOEl, 'UAE State of Energy Report 2017', 2017. [Online]. Available: http://dcce.ae/uae-state-ofenergy-report-2017/.

[9] Q. G. E. and W. C. Kahramaa, 'kahramaa Annual Statistics Report 2018', Doha, 2020. Accessed: Dec. 10, 2020. [Online]. Available: https://www.km.qa/MediaCenter/Publications/Statistics\%20Report\%202018-English.pdf.

[10] 'KSK Mahanadi Power Project', Global Energy Monitor. https://www.gem.wiki/KSK_Mahanadi_Power_Project.

[11] UPRVUNL, 'On Going Projects', Uttar Pradesh Rajya Vidyut Utpadan Nigam Limited. https://uprvunl.org/uprvunl/en/post/on-going-projects (accessed Dec. 15, 2020).

[12] TANGEDCO, 'Power Projects', Tamil Nadu Generation and Distribution Corporation Limited. https://www.tangedco.gov.in/power-projects.html (accessed Dec. 15, 2020).

[13] 'Barh I power station', Global Energy Monitor, Dec. 25, 2019. https://www.gem.wiki/Barh_I_power_station (accessed Dec. 15, 2020). 
[14] 'Darlipalli Super Thermal Power Station', Global Energy Monitor Wiki.

https://www.gem.wiki/Darlipalli_Super_Thermal_Power_Station.

[15] TNN, 'Pandemic delays NTPC's Karanpura mega project in Chatra', The Times of India, Sep. 09, 2020.

[16] 'Ennore SEZ Super Critical Thermal Power Project (Vayalur)', Global Energy Monitor. .

[17] R. Prasad, 'BHEL beats L\&T, Doosan to win 2,400-mw project', The Economic Times, Jul. 18, 2017.

[18] PTI, 'NLC begins construction work for 1980-MW project in UP', ET EnergyWorld, Oct. 21, 2020.

[19] 'Jawaharpur Super Thermal Power Station', NS Energy.

[20] 'Godda Ultra Supercritical Thermal Power Project, India', NS Energy.

[21] 'Bhadradri Thermal Power Station, Telangana', NS Energy.

[22] TNN, 'NTPC projects to bring more power to UP by 2017', The Times of India, Feb. 19, 2015.

[23] 'Nabinagar Super Thermal Power Project', NS Energy.

[24] ET Bureau, 'NTPC's total installed power generation capacity reaches 62,910 MW', ET Energyworld, Jul. 21, 2020.

[25] TNN, 'Scindia lays foundation stone of NTPC super thermal power project at Gadarwara', The Times of India, Feb. 20, 2014.

[26] B. Sivakumar, 'Tamil Nadu to get 1st 800MW thermal plant', The Times of India, Feb. 01, 2017.

[27] APPDCL, ' 1 X 800 MW SRI DAMODARAM SANJEEVAIAH THERMAL POWER STATION (STAGE -II)', Andhra Pradesh Power Development Company LTD, Jul. 2012. [Online]. Available: http://environmentclearance.nic.in/writereaddata/form1/31_SDSTPS\%20stage2\%20MoEF\%20application.pdf.

[28] 'Telangana Super-Thermal Power Project, Telangana, India', NS Energy.

[29] V. S. Rawat, 'Akhilesh cabinet okays 3 thermal power units totalling 1,980 mw', Business Standard India, Jul. $22,2014$.

[30] PTI, 'BHEL begins civil works at 660-MW Sagardighi thermal power plant in West Bengal', The Economic Times, Aug. 07, 2020.

[31] D. Sengupta, 'Odisha government clears NTPC's Talcher project', The Economic Times, Jan. 09, 2020.

[32] 'Udangudi Coal-fired Power project, Tamil Nadu, India', NS Energy.

[33] NPCIL, 'Projects Under Construction', Nuclear Power Corporation of India Limited.

https://npcil.nic.in/content/297_1_ProjectConstructionStatus.aspx (accessed Dec. 15, 2020).

[34] CEA, 'National Electricity Plan Volume I (Generation)', Central Electricity Authority, India, 2018.

[35] 'Kakrapar 3 achieves first criticality', World Nuclear News, Jul. 22, 2020. https://www.world-nuclearnews.org/Articles/Kakrapar-3-achieves-first-criticality (accessed Dec. 15, 2020).

[36] 'First phase Gorakhpur Haryana Atomic Power Plant expected to be completed in 2025', Business Standard India, Jan. 02, 2019. 
[37] 'India's first Prototype Fast Breeder Reactor in final stages of commissioning', The New Indian Express, Oct. 30, 2020.

[38] E. N. E. C. ENEC, 'Emirates Nuclear Energy Corporation', 2019. https://www.enec.gov.ae/ (accessed Oct. 01, 2019).

[39] W. N. A. WNA, 'Nuclear Power in Saudi Arabia', 2020. https://www.world-nuclear.org/information-library/countryprofiles/countries-o-s/saudi-arabia.aspx (accessed Dec. 11, 2020).

[40] I. R. E. A. IRENA, 'Renewable energy market analysis: GCC 2019', Abu Dhabi, 2019. [Online]. Available: https://www.irena.org/-/media/Files/IRENA/Agency/Publication/2019/Jan/IRENA_Market_Analysis_GCC_2019.pdf.

[41] MOSPI, 'Energy Statistics 2020', Indian Ministry of Statistics and Program Implementation, 2020. [Online]. Available: http://mospi.nic.in/sites/default/files/publication_reports/ES_2020_240420m.pdf.

[42] MoP, 'National Electricity Plan Vol. II Transmission', Indian Ministry of Power, 2018. [Online]. Available: https://powermin.nic.in/sites/default/files/uploads/NEP-Trans1.pdf.

[43] G. C. C. I. A. (GCCIA) GCCIA, 'Gulf Cooperation Council Interconnection Authority (GCCIA)', 2015. https://www.gccia.com.sa/ (accessed Dec. 11, 2020).

[44] A. Al Mohaisen, 'INTERCONNECTION PROJECT PHASE - I STATUS \& DEVELOPMENTS', Accessed: Dec. 11, 2020. [Online]. Available: https://www.gccia.com.sa/Data/PressRelease/Press_4.pdf.

[45] CEA, 'Long Term Electricity Demand Forecasting Report', Central Electricity Authority, India, 2019. Accessed: Mar. 08, 2021. [Online]. Available: https://cea.nic.in/power-survey-load-forecasting-division/?lang=en.

[46] PTI, 'IMF Projects 11.5\% GDP Growth For India In 2021', BloombergQuint, Jan. 26, 2021. https://www.bloombergquint.com/economy-finance/imf-projects-impressive-11-5-per-cent-growth-rate-for-india-in-2021 (accessed Mar. 08, 2021).

[47] V. Dhoot, 'GDP likely to contract by 7.7\% in 2020-21, says Govt.', The Hindu, New Delhi, Jan. 07, 2021.

[48] CEA, 'Load Generation Balance Report 2020-21', Central Electricity Authority, India, 2020. [Online]. Available: https://cea.nic.in/l-g-b-r-report/?lang=en.

[49] A. D. T. and D. C. TRANSCO, '2017 Electricity Seven Year Planning Statement (2018-2024)', 2017. [Online]. Available: http://www.transco.ae/media/pdf/2017\%20Electricity\%20Seven\%20Year\%20Planning\%20Statement_Main\%20Report.pdf.

[50] Y. Almulla, Gulf Cooperation Council (GCC) countries 2040 energy scenario for electricity generation and water desalination. 2015.

[51] D. E. \&amp; W. A. DEWA, 'Dubai Electricity \&amp; Water Authority (DEWA) | Home', 2019. https://www.dewa.gov.ae/en (accessed Oct. 01, 2019).

[52] S. E. and W. A. SEWA, 'SEWA | Home', 2019. https://www.sewa.gov.ae/en/ (accessed Oct. 01, 2019).

[53] F. E. and W. A. FEWA, 'Federal Electricity \& Water Authority', 2019. https://fewa.gov.ae/en/Pages/default.aspx (accessed Oct. 01, 2019).

[54] Q. E. and W. C. QEWC, 'QEWC - Annual Report 2019', Doha, 2019. Accessed: Dec. 11, 2020. [Online]. Available: https://www.qewc.com/qewc/en/?wpdmdl=7720. 
[55] O. P. A. W. P. C. OPWR, 'OPWR - Annual Report 2019', Muscat, 2019. Accessed: Dec. 11, 2020. [Online]. Available: https://omanpwp.om/PDF/OPWP-AR-2019-ENG-A4.pdf.

[56] M. of E. and W.-K. MEW, 'Electrical Energy - Statistical Year Book 2018', Kuwait, 2019. Accessed: Dec. 11, 2020. [Online]. Available: https://www.mew.gov.kw/media/2xblxq1v/engery-2018.pdf.

[57] E. and W. A.-B. EWA, 'EWA Statistics 2018', Manama, 2019. Accessed: Dec. 11, 2020. [Online]. Available: http://www.ewa.bh/en/AboutUs/AnnualReport/statistics\%202018.pdf.

[58] IEA-ETSAP, 'Coal-Fired Power', IEA-ETSAP, Apr. 2010. [Online]. Available: https://www.iea-etsap.org/ETechDS/PDF/E01-coal-fired-power-GS-AD-gct_FINAL.pdf.

[59] IEA-ETSAP, 'Gas-Fired Power', IEA-ETSAP, Apr. 2010. [Online]. Available: https://iea-etsap.org/E-TechDS/PDF/E02gas_fired_power-GS-AD-gct_FINAL.pdf.

[60] KTH-dESA, 'OSeMOSYS - The Electricity Model Base for Africa (TEMBA)'. http://www.osemosys.org/temba-theelectricity-model-base-for-africa.html.

[61] IEA-ETSAP, 'Nuclear Power', IEA-ETSAP, Apr. 2010. [Online]. Available: https://iea-etsap.org/E-TechDS/PDF/E03Nuclear-Power-GS-AD-gct.pdf.

[62] IEA-ETSAP, 'Hydropower', IEA-ETSAP. [Online]. Available: https://iea-etsap.org/E-TechDS/PDF/E06-hydropower-GSgct_ADfina_gs.pdf.

[63] IEA-ETSAP, 'Biomass for Heat and Power', IEA-ETSAP, Apr. 2010. [Online]. Available: http://www.iea-etsap.org/ETechDS/PDF/E05-BiomassforHP-GS-AD-gct.pdf.

[64] Office of Energy Efficiency \& Renewable Energy, 'Geothermal FAQs', Energy.gov. https://www.energy.gov/eere/geothermal/geothermal-faqs (accessed Dec. 15, 2020).

[65] S. Naderi, M. Banifateme, O. Pourali, A. Behbahaninia, I. MacGill, and G. Pignatta, 'Accurate capacity factor calculation of waste-to-energy power plants based on availability analysis and design/off-design performance', Journal of Cleaner Production, vol. 275, p. 123167, Dec. 2020, doi: 10.1016/j.jclepro.2020.123167.

[66] M. Brinkerink and P. Deane, 'PLEXOS-World 2015'. Harvard Dataverse, Dec. 11, 2020, doi: 10.7910/DVN/CBYXBY.

[67] K. A. P. S. and R. C. KAPSARC, 'KAPSARC Energy Data Portal', 2020. https://www.kapsarc.org/research/data-tools/ (accessed Dec. 11, 2020).

[68] O. P. A. W. P. C. OPWR, 'OPWR - Annual Report 2017', Muscat, 2017. Accessed: Dec. 11, 2020. [Online]. Available: https://www.omanpwp.om/PDF/OPWP\%20Annual\%20Report\%202017_ENG_FINAL.pdf.

\section{Figures}




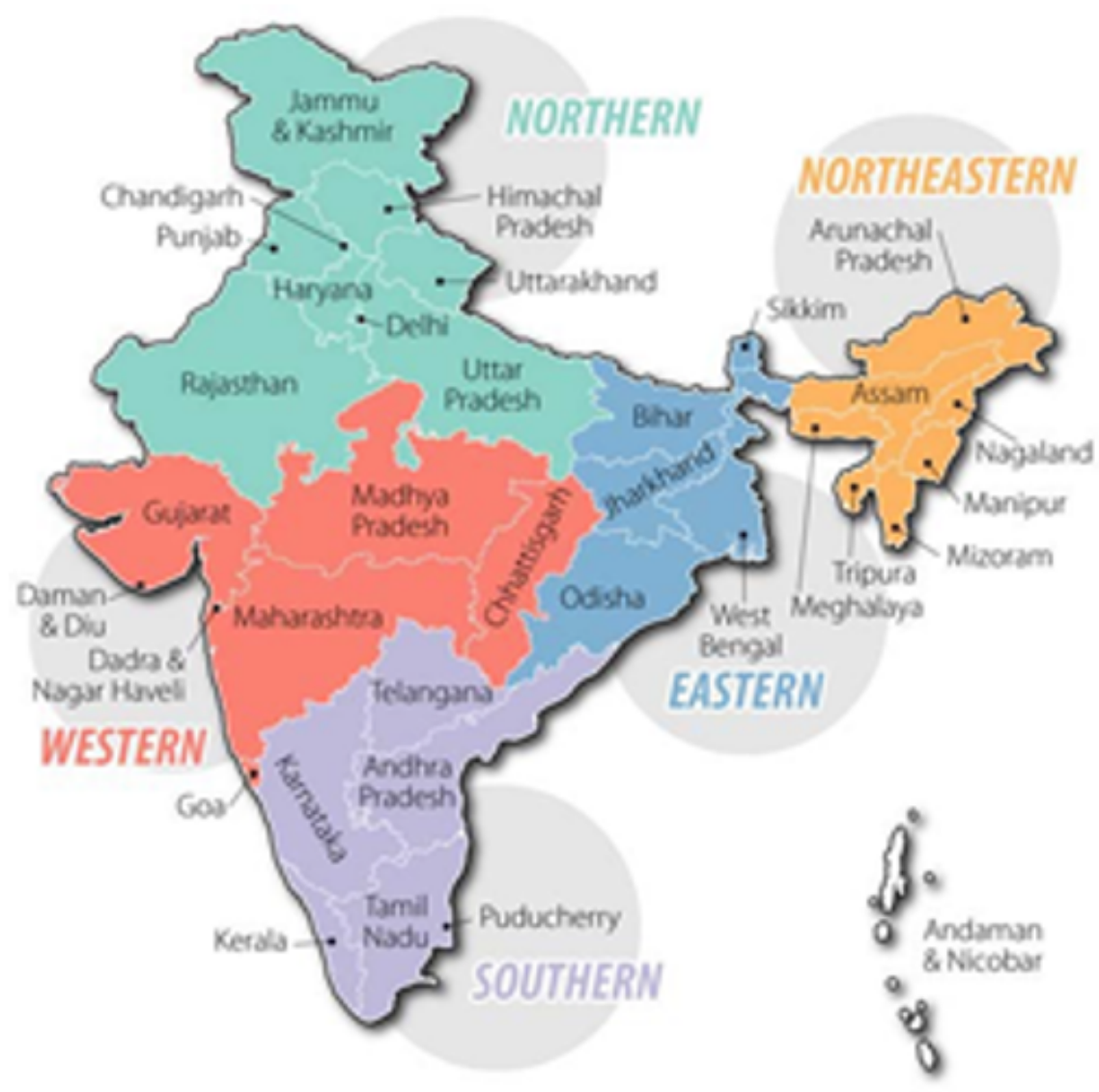

Figure 1

India's regional electricity grids [1] Note: The designations employed and the presentation of the material on this map do not imply the expression of any opinion whatsoever on the part of Research Square concerning the legal status of any country, territory, city or area or of its authorities, or concerning the delimitation of its frontiers or boundaries. This map has been provided by the authors. 


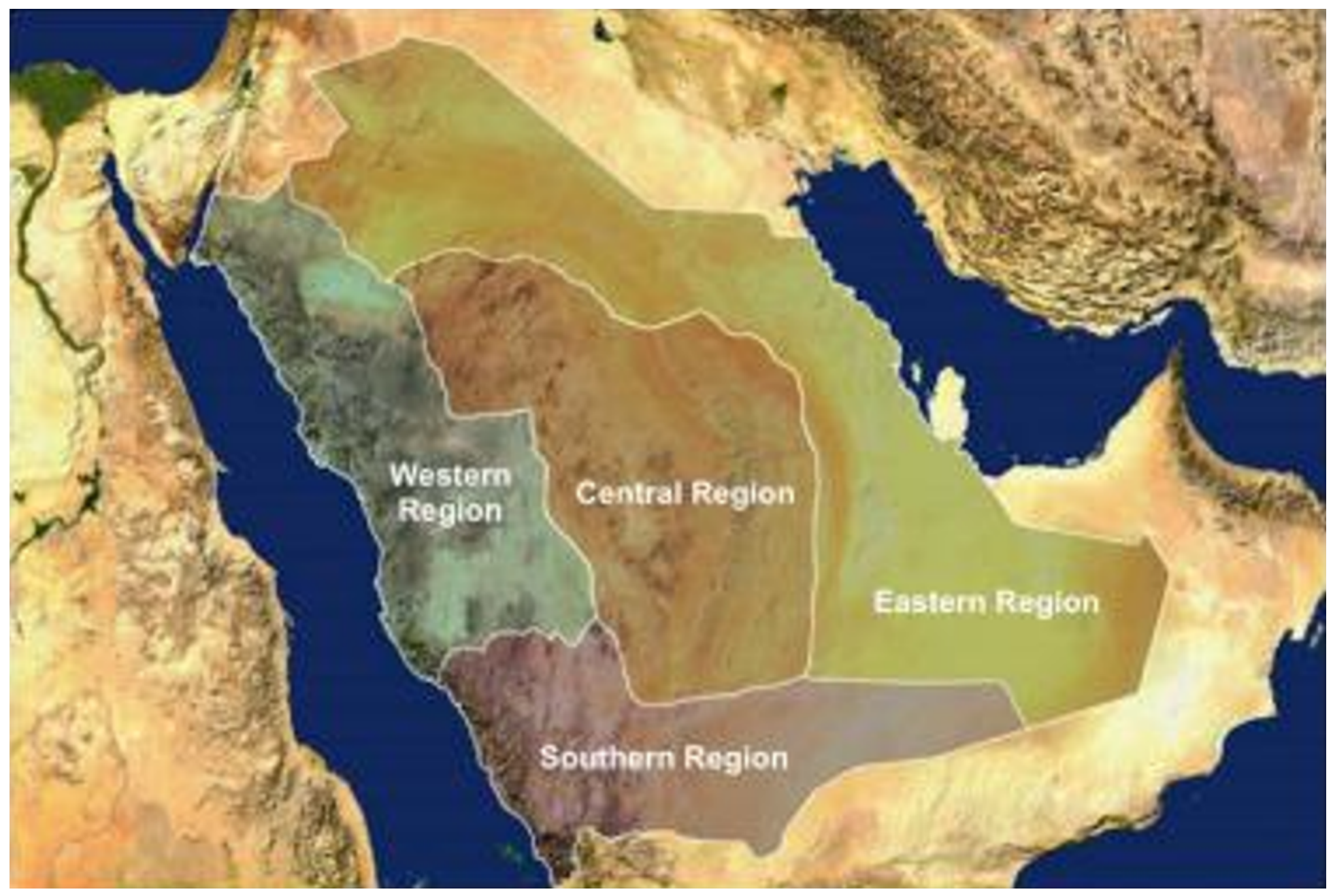

Figure 2

Saudi Arabia regions [2] Note: The designations employed and the presentation of the material on this map do not imply the expression of any opinion whatsoever on the part of Research Square concerning the legal status of any country, territory, city or area or of its authorities, or concerning the delimitation of its frontiers or boundaries. This map has been provided by the authors. 